\title{
Possible radiologic renal signs of COVID-19
}

\author{
Isil Basara Akin ${ }^{1}\left[\right.$ Canan Altay $^{1} \cdot$ Oya Eren Kutsoylu $^{2} \cdot$ Mustafa Secil $^{1}$
}

Received: 15 June 2020 / Revised: 30 June 2020 / Accepted: 18 July 2020 / Published online: 28 July 2020

(c) Springer Science+Business Media, LLC, part of Springer Nature 2020

\begin{abstract}
Coronavirus disease 2019 (COVID-19) has caused a huge outbreak worldwide. Patients infected with COVID-19 most commonly present with respiratory tract symptoms and pneumonia. Gastrointestinal symptoms can also occur in these patients. Renal involvement presenting as acute renal infarct and/or acute kidney injury has been described in literature; however, these typically occur in patients with severe COVID-19. To the best of our knowledge, there are no reported cases describing abnormal renal imaging findings in a patient with only mild form of COVID-19. We, therefore, report a case of a patient with mild COVID-19 presenting with acute abdominal pain and acute renal infarct.
\end{abstract}

Keywords Computed tomography $\cdot$ Coronavirus disease $\cdot$ Renal infarct

\section{Introduction}

Coronavirus disease 2019 (COVID-19) has caused a huge outbreak worldwide. Currently, the number of infected patients has exceeded 9,000,000. Patients with COVID-19 most commonly present with respiratory tract symptoms and pneumonia [1,2]. Disease is classified as severe in patients who are hypoxic and require mechanical ventilation [3].

Abdominal symptoms can also occur in COVID-19 patients. Recent data from Wuhan revealed that gastrointestinal symptoms such as diarrhea were present in $79 \%$ of COVID-19 infected patients [4]. Although, clinical presentation of patients with abdominal symptoms has been previously reported, to date there are only a few studies describing the abdominal imaging findings in these patients. In a recent study, Bhayana et al. described bowel abnormalities and cholestasis as the most common abdominal imaging findings [5]. Additionally, apart from gastrointestinal findings, renal pathologies including renal infarct have also been reported [6-12]. However, renal findings are typically seen in patients with severe COVID-19 disease. To the best of

Isil Basara Akin

slbasara@yahoo.com

1 Department of Radiology, Dokuz Eylul University School of Medicine, Izmir, Turkey

2 Department of Infectious Diseases and Clinical Microbiology, Dokuz Eylul University School of Medicine, Izmir, Turkey our knowledge, there are no published reports describing abnormal renal findings in a patient with mild form of the COVID-19 disease.

Here we present a case of mild COVID-19 with abnormal renal findings on computed tomography (CT).

\section{Case report}

A 48-year-old male patient presented to our institution with symptoms of high fever $\left(38^{\circ} \mathrm{C}\right)$ and abdominal pain for 4 days. He had no respiratory tract symptoms and no abnormal findings on his physical examination and reported no history of respiratory problems. His laboratory workup revealed leukopenia $\left(3.8 \times 10^{-3} \mu / \mathrm{L}\right)$ and lymphopenia $\left(1.2 \times 10^{-3} \mu / \mathrm{L}\right)$. Additionally, the following parameters were abnormally elevated: D-dimer $(0.7 \mu \mathrm{g} / \mathrm{mL})$, lactate dehydrogenase $(567 \mathrm{U} / \mathrm{L})$, alanine aminotransferase $(59 \mathrm{U} / \mathrm{L})$, ferritin $(577.8 \mathrm{ng} / \mathrm{mL})$ and CRP $(138.2 \mathrm{mg} / \mathrm{L})$. Creatinine and e-GFR were within normal limits. The patient was sent to radiology department for further evaluation with contrast enhanced chest and abdominal CT exams. Chest CT demonstrated patchy, ground-glass perilobular opacities with reticular densities at the peripheral portions of both lungs (Fig. 1). Additionally, reverse halo sign was present in the posterior segment of right upper lobe. The CT findings were described as possible COVID-19 findings. There were no signs of pulmonary arterial embolism. On the abdominal CT, right kidney showed patchy, sharply demarcated 

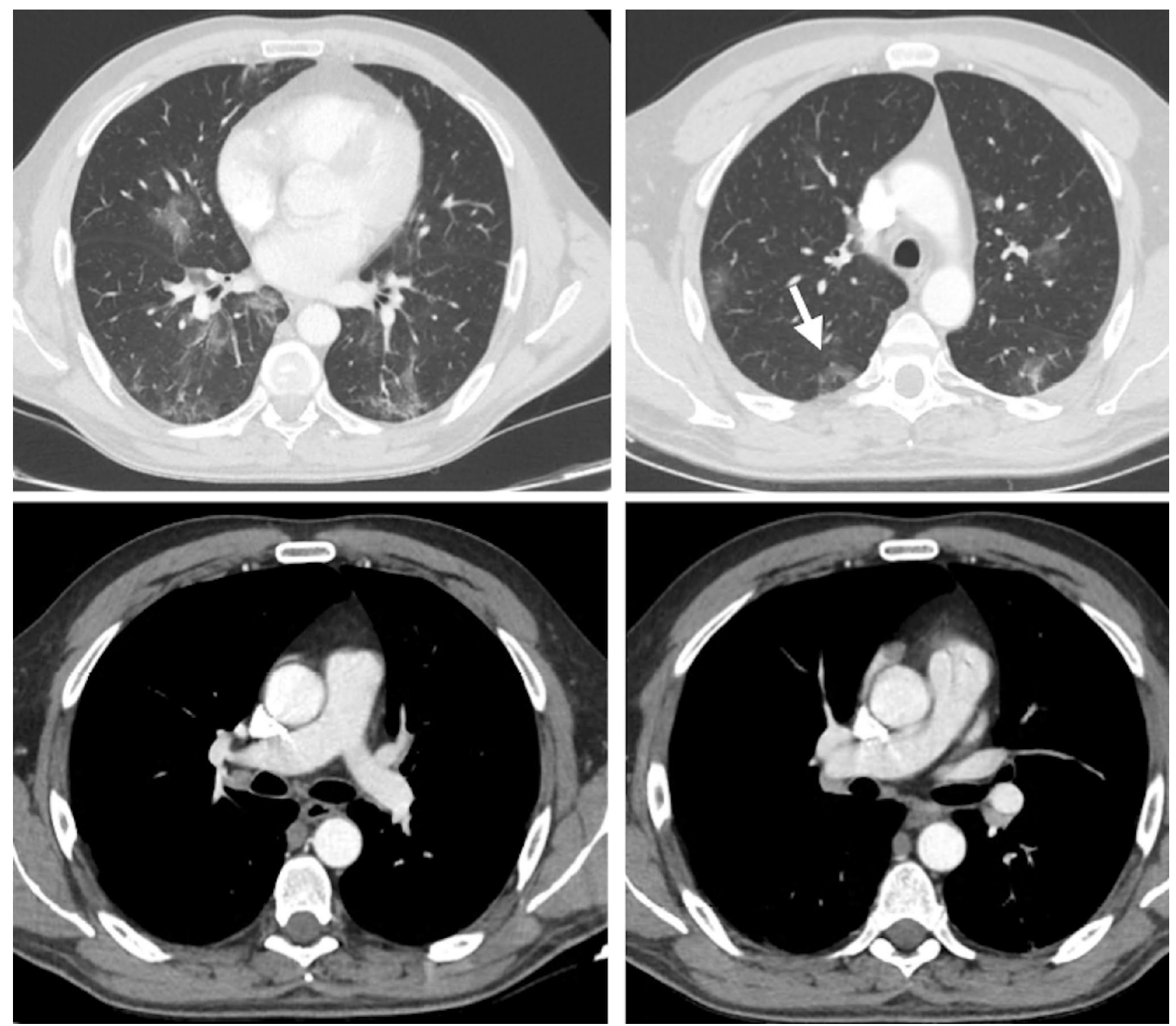

Fig. 1 Peripheral patchy ground-glass peribronchovascular infiltrations are present with reticular densities in both pulmonary lobes. Reverse halo sign is seen at right lung, upper-posterior segment (arrow). There is no sign of thromboembolism in the major pulmonary arteries

hypoenhancing and nonenhancing areas in the anterior part of lower pole with increased heterogeneous linear densities in peri and pararenal areas (Fig. 2). No other abnormalities were seen on the abdominal CT. As a result of clinical and abdominal imaging findings, segmental renal infarct was the patient's leading diagnosis and he was admitted to our hospital. In the light of chest and renal findings, polymerase chain reaction (PCR) test from nasal swab for COVID-19 was ordered and urine analysis with urine culture tests was performed. PCR test returned positive for COVID-19 infection. No abnormalities were detected on urinalysis and urine culture was negative. Patient did not develop any respiratory symptoms throughout his hospital course and did not require admission to the intensive care unit. He was treated with hydroxychloroquine and oseltamivir, according to COVID19 protocol of our community's health ministry. In addition, given the renal findings on CT and the diagnosis of renal infarct, patient was given low-molecular-weight heparin. After fifth day of the medical treatment, the fever dropped, and his abdominal pain decreased. On the 9th and 11th days of hospitalization, nasal swab for PCR tests were repeated and the results had turned to negative on both of those tests. Besides, all of his abdominal symptoms had resolved. Therefore, he was discharged, and sent to quarantine at home. 

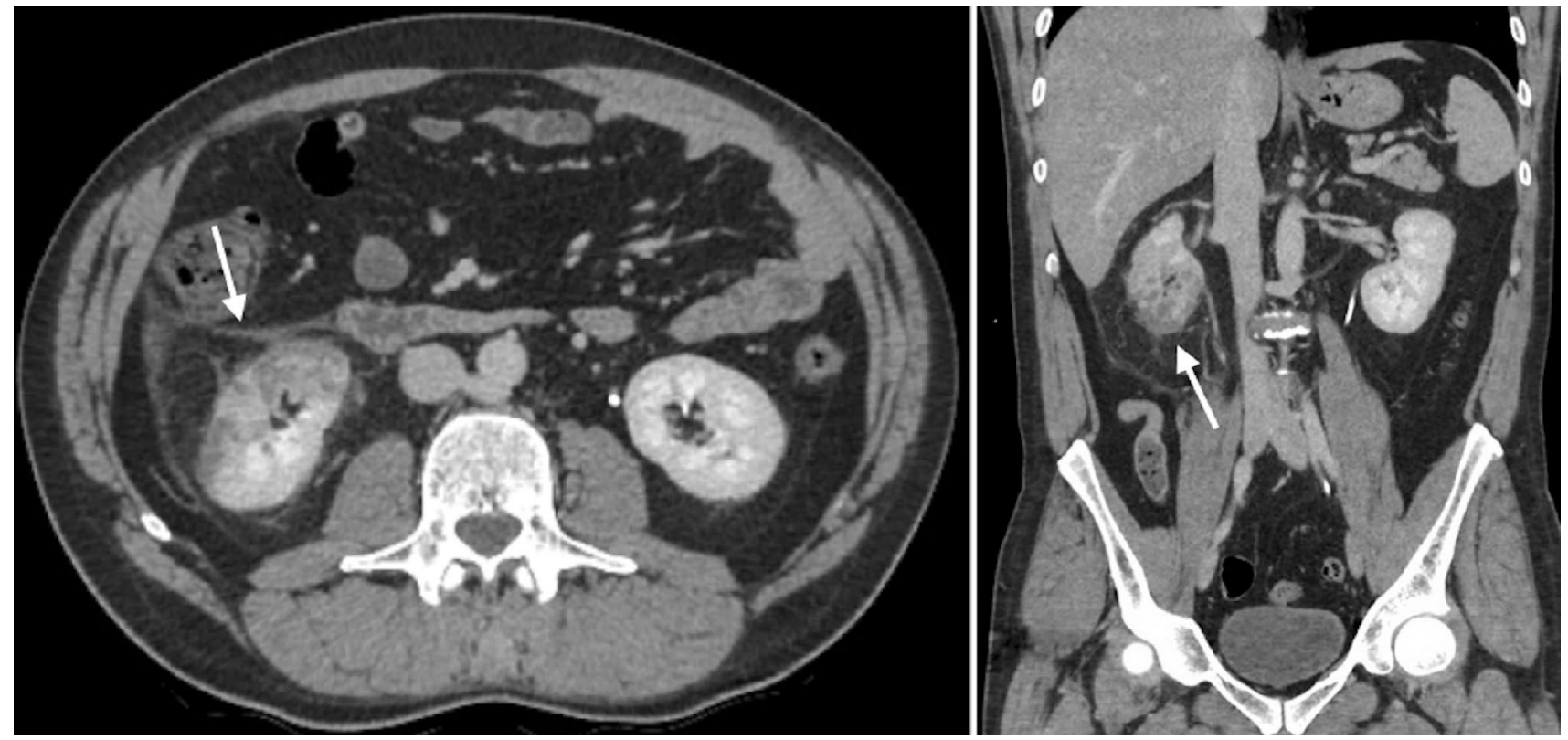

Fig. 2 Right kidney demonstrates sharply demarcated heterogeneous area containing nonenhancing and hypoenhancing portions (arrow). Significant perinephric stranding is also present

\section{Discussion}

Abdominal symptoms in patients with COVID-19 mostly commonly involve gastrointestinal system and can occur in $16 \%-79 \%$ of patients $[4,5,13]$. Cross-sectional abdominal imaging is not a routine application in COVID-19 patients and is usually performed when the patient presents with abdominal pain and/or sepsis. The most common abdominal findings on imaging has been reported as bowel wall thickening and cholestasis [5].

Renal abnormalities in patients with COVID-19 have been previously described. These renal pathologies include COVID-19 associated acute kidney injury (AKI) [11] and there have been a few reports describing renal infarcts due to severe hypercoagulability state which can occur in these patients [5-9]. Presence of AKI in patients with COVID-19 was found to be a rare complication [11]. In a cohort from China with 1099 COVID-19 patients, the rate of AKI was $0.5 \%$ [14]. Possible mechanisms of AKI were described in three different ways as cytokine damage, systemic effects, and organ crosstalk [11]. Su et al. reported postmortem renal histopathological analysis of 26 patients, which demonstrated a wide range of histopathological findings [12]. The most common pathology was acute tubular necrosis due to endothelial damage causing microvascular lumen occlusion. They described that their results had been dependent on the possible kidney cells' infections with COVID-19. Thus, they concluded that even in patients with less severe COVID-19 infections histopathologic changes might be also seen [12]. In patients with severe COVID-19 pneumonia, hypercoagulability related pathologies were reported. In these patients, the level of D-dimer was found to be in high levels $[15,16]$. Spieza et al. observed that their study population had showed severe hypercoagulopathy and they had linked this result to increased fibrinogen levels and excessive fibrin polymerization caused by COVID-19 disease [13]. The authors concluded that microcirculation thrombosis, arterial complications and major venous thromboembolic pathologies might be seen in patients with massive D-dimer levels and prolonged intensive care unit hospitalization with long mechanical ventilation treatment [15].

In our case, COVID-19 infection was classified as a mild form of the disease due to clinical and radiological findings. Renal findings were unexpected and were diagnosed incidentally on CT. Except for the abdominal pain, there were no specific initial clinical or laboratory findings to suggest presence of any intra-abdominal pathology. The findings on abdominal CT were thought to represent acute renal infarct and due to the abnormal lung findings patient was diagnosed with COVID-19 infection. Given the elevated D-dimer values, we hypothesized that the renal findings might have occurred either due to the COVID-19 related mechanisms causing AKI and/or underlying hypercoagulopathy. To date, there have only been few reported cases describing renal imaging findings and all of those cases were dependent on the underlying severe hypercoagulopathy [6-9]. Apart from these cases, our case is the first in the literature with abnormal renal radiological findings occurring in a patient with only mild COVID-19 and no signs of thromboembolic findings in any other organs. We strongly believe that the 
presenting symptom of abdominal pain in our patient was caused by renal infarct due to possible endothelial damage causing from microvascular damage related to the virus.

COVID-19 is a disease with frequent pneumonia and gastrointestinal findings, but renal findings have still not been widely described. In our case, the discordance between radiologic and laboratory findings might have been the result of renal microvascular damage and infarct related to COVID-19 disease. Radiologic examinations should be performed in patients even with insignificant abdominal clinical symptoms. This way the additional abdominal findings may be detected, and unknown aspects of COVID-19 could be diagnosed.

Funding There is no funding of this article.

\section{Compliance with ethical standards}

Conflict of interest The authors declared that there is no conflict of interest.

Ethical approval All procedures performed in studies involving human participants were in accordance with the ethical standards of the institutional and/or national research committee and with the 1964 Helsinki declaration and its later amendments or comparable ethical standards.

Informed consent Informed consent was obtained from all individual participants included in the study.

\section{References}

1. Zhu N, Zhang D, Wang W, et al (2020) A novel coronavirus from patients with pneumonia in China, 2019. N Engl J Med 382:727733. https://doi.org/10.1056/NEJMoa2001017.

2. Wu, Z, McGoogan, JM (2020) Characteristics of and Important Lessons From the Coronavirus Disease 2019 (COVID-19) Outbreak in China: Summary of a Report of 72314 Cases From the Chinese Center for Disease Control and Prevention. JAMA, https ://doi.org/10.1001/jama.2020.2648. Advance online publication. https://doi.org/10.1001/jama.2020.2648

3. General Office of National Health Commission. the Diagnosis and Treatment of Pneumonia Infected by Novel Coronavirus (5th Trial Edition). https://www.gov.cn/zhengce/zhengceku/2020-02/05/ content_5474791.htm.

4. Tian Y, Rong L, Nian W, He Y (2020) Review article: gastrointestinal features in COVID-19 and the possibility of faecal transmission. Alimentary pharmacology \& therapeutics, 51:843851. https://doi.org/10.1111/apt.15731.

5. Bhayana R, Som A, Li MD, et al (2020) Abdominal Imaging Findings in COVID-19: Preliminary Observations. Radiology, 201908. Advance online publication. https://doi.org/10.1148/radiol.20202 01908.

6. Lushina N, Kuo JS, Shaikh HA (2020) Pulmonary, Cerebral, and Renal Thromboembolic Disease Associated with COVID19 Infection. Radiology, 201623. https://doi.org/10.1148/radio 1.2020201623.

7. Xu JJ, Samaha D, Mondhe S, Massicotte-Azarniouch D, Knoll G, Ruzicka M (2020) Renal Infarct in a COVID-19 Positive Kidney-Pancreas Transplant Recipient. Am J Transplant https://doi. org/10.1111/ajt.16089.

8. Farkash EA, Wilson AM, Jentzen JM (2020) Ultrastructural Evidence for Direct Renal Infection with SARS-CoV-2. J Am Soc Nephrol. https://doi.org/10.1681/ASN.2020040432.

9. Tascón GC, Chiriboga DES, Ramos RL, et al (2020) Infarto renal en paciente con infección activa por COVID -19 [Renal infarction in a patient with active COVID -19 infection]. Nefrologia. https:// doi.org/10.1016/j.nefro.2020.04.008.

10. Fanelli V, Fiorentino M, Cantaluppi V, Gesualdo L, Stallone G, Ronco C, Castellano G (2020) Acute kidney injury in SARSCoV-2 infected patients. Critical Care (London, England), 24:155. https://doi.org/10.1186/s13054-020-02872-z.

11. Ronco C, Reis T (2020) Kidney involvement in COVID-19 and rationale for extracorporeal therapies. Nat Rev Nephrol. 16:308-310. https://doi.org/10.1038/s41581-020-0284-7.

12. Su H, Yang M, Wan C, et al (2020) Renal histopathological analysis of 26 postmortem findings of patients with COVID19 in China. Kidney Int. S0085-2538(20)30369-0. https://doi. org/10.1016/j.kint.2020.04.003.

13. Luo S, Zhang X, Xu H (2020) Don't Overlook Digestive Symptoms in Patients With 2019 Novel Coronavirus Disease (COVID19). Clin Gastroenterol Hepatol. 18:1636-1637. https://doi. org/10.1016/j.cgh.2020.03.043.

14. Guan WJ, Ni ZY, Hu Y, et al (2020) Clinical Characteristics of Coronavirus Disease 2019 in China. N Engl J Med. 382:1708-1720. https://doi.org/10.1056/NEJMoa2002032.

15. Spiezia L, Boscolo A, Poletto F, et al (2020) COVID-19-Related Severe Hypercoagulability in Patients Admitted to Intensive Care Unit for Acute Respiratory Failure. Thromb Haemost. 120:998-1000. https://doi.org/10.1055/s-0040-1710018.

16. Tang N, Li D, Wang X, Sun Z (2020) Abnormal coagulation parameters are associated with poor prognosis in patients with novel coronavirus pneumonia. J Thromb Haemost 18:844-847. https://doi.org/10.1111/jth.14768

Publisher's Note Springer Nature remains neutral with regard to jurisdictional claims in published maps and institutional affiliations. 